\title{
Application of Concatenated Codes in the IEEE802.11b Channel Coding
}

\author{
Zengyou Sun \\ College of Computer Engineering \\ Northeast Dianli University \\ Jilin, China \\ E-mail: sunzengyou@163.com
}

\author{
Huanhuan Li \\ College of Computer Engineering \\ Northeast Dianli University \\ Jilin, China \\ E-mail: lhh1989nedu@163.com
}

\begin{abstract}
According to the problem of the data transmission system of IEEE802.11b in the presence of random noise and burst interference which make the receiver error, we use the channel coding scheme of concatenated codes, RS codes and convolution codes concatenated, as a block code and non-block code concatenated coding, the outer code with RS codes, the inner code with convolution codes. And we use MATLAB to simulate the channel error correction performance of the concatenated code in the additive Gauss white noise channel. The results show that in the case of low SNR, RS+convolution concatenated codes can make the system BER down to $10^{-5}$ below(including $10^{-5}$ ), it is a kind of efficient error correcting code, and has high practical value in engineering.
\end{abstract}

Keywords- convolution codes; RS codes; concatenated codes; IEEE802.11b

\section{INTRODUCTION}

The IEEE802.11b standard is expanded based on the IEEE802.11 standard, it uses the complementary code keying (CCK) modulation technology, so the data transmission rate can be up to $11 \mathrm{Mbps}$ in the $2.4 \mathrm{GHz}$ ISM band, which has greatly promoted the development of wireless local area network (WLAN). We know in the condition of no multi-path channel and high SNR, CCK system can achieve better performance, while in the low SNR or the surge in the number of co-channel users, the bit error rate(BER) is rapidly increasing seriously and affect the quality of communication[1]. This problem has become a bottleneck restricting the expansion of application fields of CCK technology. This paper seeks a kind of error control code with high efficiency, high gain, good performance of error correction coding, encoding and decoding method is relatively simple and small amount of computation decoding, then combine it with CCK modulation and demodulation technology to effectively inhibit the error rate increase and realize the high quality digital communication of CCK system. RS codes and convolution code concatenated to complete channel coding, which is the simple way to achieve the above purpose.

\section{CHANNEL CODING}

\section{A. Concatenated Codes}

The channel coding theorem states that, with the increase of code length $n$, decoding error probability exponentially close to zero. Therefore, in order to make the code effectively, we must use the long codes, but with the code length increases, the number of required correction in a code group increases, the decoder complexity and computational cost also increases accordingly, so that is difficult to achieve[2]. To solve the contradictions between the error correction performance and equipment complexity, Forney proposed the concept of concatenated code in 1966, the process of the preparation of long codes in several stages, usually divided into two levels; each level sub-code is taken from different domains and then connected in series, with a strong ability to remedy burst and random errors.

The complexity of the concatenated codes equipment mainly depends on the decoder; it can be proved that its complexity does not increase exponentially with the increase of code length, but increase with a small power. Therefore, from the perspective of the decoding complexity, concatenated code is much better than a level code.

\section{B. RS+Convolution Concatenated Codes in IEEE802.11b Channel}

The RS codes and convolution codes concatenated, as a block code and non-block code concatenated coding, the outer code with RS codes, the inner code using convolution codes with coding efficiency $1 / \mathrm{n}$ and Viterbi decoding, in the wireless communication, especially in deep space communication, concatenated codes obtain better effect.

When random errors are more and accompanied by bust errors, as the outer code, RS codes is able to make up for the inadequacy of convolution codes and easily correct the error which the convolution codes cannot correct[3], that is because the residual errors of convolution codes is usually a group. Such cooperation can give full play to all levels of code error correction performance. Especially in the channel under the condition of poor quality, appropriate decoding method selection of RS codes and convolution codes, the concatenated codes can well control the complexity of system and achieve better performance of error correction. The improved IEEE802.11b channel coding system is shown in figure 1. 


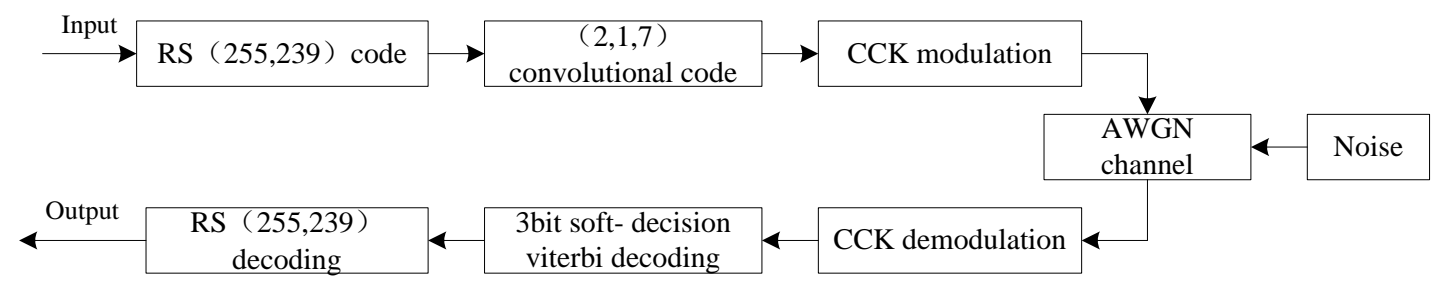

Figure 1. The improved IEEE802.11b channel coding system

\section{PERformance ANALysis OF RS+CONVOLUTION CONCATENATED CODES}

\section{A. Parameters Selection of RS Codes and the Process of Its Encoding and Decoding}

RS codes is used to correct $t$ symbol random errors, but also suitable for burst errors correcting. The RS codes which can correct $t$ symbol random errors has the following parameters: the bits of each symbol is $m$; each symbol can be regarded as an element in the finite field $G F\left(2^{m}\right)$, its code length is $n=2^{m}-1$; information segment is $k$ bits: $k=n-2 t$; symbols bits to supervise is $n-k=2 t$; the minimum code distance of symbols is $d_{0}=2 t+1=d_{\min }$. Generally, RS codes choose $G F\left(2^{4}\right)$ and $G F\left(2^{8}\right)$, the greater $m$ is, the stronger the ability of error correction will be. Considering the complexity of the algorithm and error correction ability, we choose $G F\left(2^{8}\right) . G F\left(2^{8}\right)$ domain corresponding to 8 bits binary number, that is, a byte, so this makes it convenient for computer processing, eliminates the problem of other domains or codes require a lot of bits processing and improves the efficiency of computer processing, also increases the real-time [4].

Considering the code group of the RS codes is $G F\left(2^{m}\right)$, where $m$ is the binary number for each symbol. Make

$$
d(x)=c_{n-k} x^{n-k}+c_{n-k+1} x^{n-k+1}+\cdots c_{n-1} x^{n-1}
$$

Message polynomial;

$$
p(x)=c_{0}+c_{1} x+\cdots+c_{n-k-1} x^{n-k-1}
$$

As the supervision of polynomial, the polynomial of RS codes can be expressed as:

$$
c(x)=p(x)+d(x)
$$

The most common cyclic code is using $d(x)$ and $g(x)$ to calculate $p(x)$, namely $d(x)$ divided by $g(x)$, the result is $q(x)$, the remainder is $\gamma(x)$, so there is

$$
d(x)=q(x) g(x)+\gamma(x)
$$

Take formula (4) into formula (3), we obtain

$$
c(x)=p(x)+q(x) g(x)+\gamma(x)
$$

Let $p(x)=-\gamma(x)$, there is

$$
c(x)=q(x) g(x)
$$

Let $\alpha$ be the original element of $G F\left(2^{m}\right)$. The generation polynomial of RS codes with error correction ability of length $t$ can be expressed as

$$
g(x)=(x+\alpha)\left(x+\alpha^{2}\right) \cdots\left(x+\alpha^{2 t}\right)=\prod_{j=1}^{2 t}\left(x+\alpha^{j}\right)
$$

Therefore, the RS codes will be the above process to gain the supervision polynomial $p(x)$. The direct method of obtaining the residue divided by $G(X)$ is connected a shift register via $g(x)$.

If $c(x)$ is the transmitted codes, $r(x)$ is the received codes after transmitting, the error pattern is

$$
e(x)=r(x)+c(x)=\sum_{i=0}^{n-1}\left(r_{i}+c_{i}\right) x^{i}=\sum_{i=0}^{n-1} e_{i} x^{i}
$$

Where $x^{i}$ is the error location, $e_{i}$ is the wrong size, formula (8) can be written as $r(x)=e(x)+c(x)$, take the root of polynomial $\alpha, \alpha^{2}, \cdots, \alpha^{2 t}$ into it, we have

$$
s_{i}=r\left(\alpha^{i}\right)=c\left(\alpha^{i}\right)+e\left(\alpha^{i}\right)
$$

Then the following matrix:

$$
\left[\begin{array}{c}
s_{1} \\
s_{2} \\
\cdots \\
s_{2 t}
\end{array}\right]=\left[\begin{array}{cccc}
x_{1}{ }^{1} & x_{1}{ }^{2} & \cdots & x_{1}{ }^{t} \\
x_{2}{ }^{1} & x_{2}{ }^{2} & \cdots & x_{2}{ }^{t} \\
\cdots & \cdots & \cdots & \cdots \\
x_{2 t}{ }^{1} & x_{2 t}{ }^{2} & \cdots & x_{2 t}{ }^{t}
\end{array}\right]\left[\begin{array}{c}
e_{1} \\
e_{2} \\
\cdots \\
e_{t}
\end{array}\right]
$$

Matrix calculation can get the error location $x^{i}$ and error size $e_{i}$, this error locator polynomial of error correcting RS codes can be written as $\sigma(x)=\prod_{i=1}^{t}\left(1+\alpha_{i} x\right)$, the inverse of $\sigma(x)$ is the error position number of $e(x)$. One solution to solve the above matrix calculation is using the iterative 
algorithm, known as Berlekamp iterative algorithm. The result is

$$
e_{j}=\frac{\sum_{i=0}^{v-1} \sigma_{j i} s_{v-1}}{\sum_{i=0}^{v-1} \sigma_{j i} X_{v-1}{ }^{j}}=\frac{\sum_{i=0}^{v-1} \sigma_{j i} s_{v-i}}{X^{j} \sum_{i=0}^{v-1} \sigma_{j i} X_{v-i-1}^{j}}
$$

Then put the value of the error in the wrong place, the error correction tasks are completed.

\section{B. Parameters Selection of Convolution Codes and the Process of Encoding and Decoding}

Convolution codes is a kind of forward error codes, usually a convolution codes is referred to the $(n, k, m)$ type convolution codes, where $n$ is the output number of bits, $k$ is the input number of message bits, $m$ is the encoding memory, which means that the unit time of the input packet to be stored in the encoder, $m+1=N$ is the encoding restriction. Usually, $(n, k, m)$ is composed of $m \times k$ bits shift register and some combinational logic. If the symbols of outer RS codes are taken from $G F\left(2^{M}\right)$ domain, it will be more appropriate that convolution encoding constraint degree $m$ is equal to $M$ [5]. The inner convolution codes rate $R$ usually choose $1 / 2$ or $1 / 3$, this is because in the concatenated codes system, the requirements of the inner code BER is about $10^{-3}$, and convolution codes with $R=1 / 2$ or $1 / 3$ is easy to reach, and the decoder is easy to implement. On the other hand, if we choose a lower code rate, not only the decoder will be more complex, but also the information rate of the concatenated codes system will be too low.

The encoder of the $(2,1,7)$ convolution codes in the concatenated code scheme has 6 shift registers, its generating polynomial is:

$$
\begin{aligned}
& G_{0}(n)=x(n)+x(n-1)+x(n-2)+x(n-3)+x(n-6) \\
& G_{1}(n)=x(n)+x(n-2)+x(n-3)+x(n-5)+x(n-6)
\end{aligned}
$$

Where $x(n)$ is the input information sequence at the current moment $n, x(n-m)$ is the input information sequence at the ahead of time $m, G_{0}(n)$ and $G_{1}(n)$ are the encoded information, after the parallel to serial conversion is the output of the encoder. Thus obtained the generation polynomial matrix of $(2,1,7)$ convolution code:

$$
G(D)=\left[1+D+D^{2}+D^{3}+D^{6} \quad 1+D^{2}+D^{3}+D^{5}+D^{6}\right]
$$

Where $D$ represents the delay produced by the shift register. Because every element in the generated polynomial matrix can be expressed as a binary vector, it is called the encoder connection vector. Connection vector contains the information of connection relationship between the encoder and modulo-2 adder, and we can obtain the connection vector of the encoder from the above formula:

$$
\begin{aligned}
& G_{0}(D)=1+D+D^{2}+D^{3}+D^{6} \rightarrow g_{0}=[1111001] \\
& G_{1}(D)=1+D^{2}+D^{3}+D^{5}+D^{6} \rightarrow g_{1}=[1011011]
\end{aligned}
$$

The encoder structure of $(2,1,7)$ convolution code can be obtained directly generated polynomial matrix $G(D)$, as shown in figure 2.

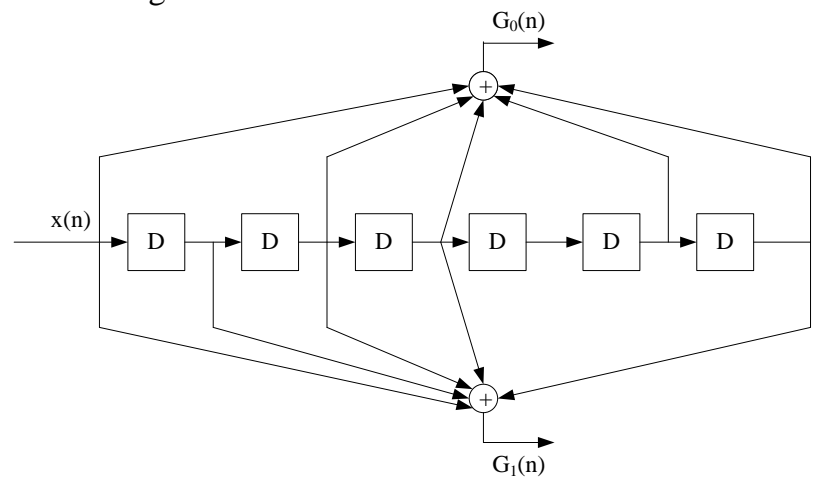

Figure 2. Coding structures of $(2,1,7)$ convolution codes

The code sequence $C$ which sends by encoder, transmitting through the discrete channel without memory, is sent into the decoder, the sequence is $R=C+E, E$ is the error sequence of the channel. The decoder is according to the received sequence $R$ and the maximum likelihood decoding criterion to find the path of the encoder through the trellis; it is the process of calculating, finding the maximum likelihood function. The likelihood function of $C_{i}$ is defined as the path metric of $C_{i}, M\left(R \mid C_{i}\right)=\log _{b} P\left(R \mid C_{i}\right)$. So there is

$$
\max _{i} \log _{b} P\left(R \mid C_{i}\right)=\max _{i} M\left(R \mid C_{i}\right) \quad i=1,2, \ldots, 2^{k L}
$$

That is the process of decoder calculating and looking for the maximum "measure" path. Where $L$ is the number of information segment used for encode.

On the BSC channel, the decoder receives the binary symbols, calculation and looking for the path with the maximum metric, is equivalent to finding a path which has the minimum Hamming distance with $R$, i.e., to find

$$
\min _{i} d\left(R, C_{i}\right) \quad i=1,2, \ldots, 2^{k L}
$$

This process is called hard decision decoding.

For the DMC channel of binary input Q-ary output, is to find the path which has the minimum soft decision distance path with $R$, this measurement is the soft decision metric distance, 
Where $R_{s}$ and $C_{i s}$ are the Q-ary representation of the sequence $R$ and $C_{i}$. This process is called soft decision decoding.

In this paper, $(2,1,7)$ convolution codes in the concatenated codes scheme uses Viterbi soft decision decoding of 3 bit quantization.

\section{Simulation Analysis}

This paper adopts the RS $(255,239)$ on the $G F\left(2^{8}\right)$ and convolution $(2,1,7)$ concatenated encoding, compared with the original data, after CCK modulation into the additive Gauss white noise channel, the received signal after CCK demodulation, RS iterative decoding and Viterbi decoding, we finally get the decoded data, compared with the original data, we can obtain the BER of the system. Under the condition of coding efficiency approximately equal, the error correction performances of the RS codes, convolution codes and RS+convolution concatenated codes based on CCK platform are analyzed and compared. The system BER curve is shown in figure 3.

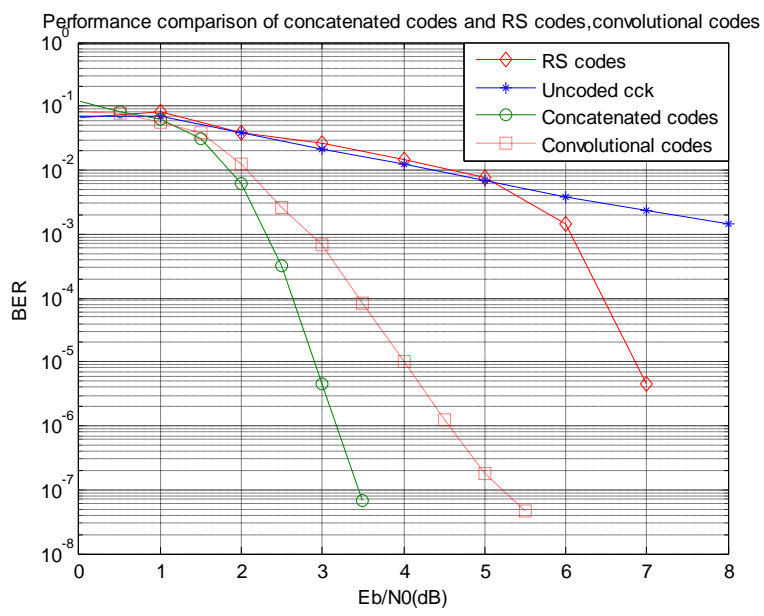

Figure 3. The system BER curve

The simulation curves show that the error correction performance of $\mathrm{RS}+$ convolution concatenated codes is higher than that of RS codes and convolution codes. The BER of the system joining the RS codes drops quickly only when SNR is greater than $6 \mathrm{~dB}$, and its performance is not good as the convolution codes and concatenated codes; the BER of the system adding convolution codes reached $10^{-5}$ when SNR $4 \mathrm{~dB}$, and dropped to $10^{-6}$ when SNR $4.5 \mathrm{~dB}$; the BER of the system adding concatenated codes reached $10^{-6}$ when SNR is $3.1 \mathrm{~dB}$, and is less than $10^{-7}$ or reached $10^{-8}$ magnitude when SNR $3.5 \mathrm{~dB}$, its decline curve is steeper than convolution codes, and the coding gain relatively to the convolution codes is also increased rapidly with the increase of SNR.

\section{CONCLUSIONS}

Through the analysis on the experiments results, after joining the concatenated codes module, the performance of IEEE 802.11b system is greatly improved than independent
RS codes and the convolution codes system. In this paper, the proposed RS $(255,239)+(2,1,7)$ convolution codes concatenated scheme, can be used in the CCK platform, and can well meet the actual system of high data transmission rate and good error correction performance requirements.

\section{REFERENCES}

[1] Yunfei Luo. CCK code modulation performance analysis and simulation[J]. Experimental science and technology, 2010,8(4):8-11.

[2] Shannon C E. The Mathematical Theory of Communications[J]. Bell System Technical Journal, 1948, vol.27:379-423,623-656.

[3] Mei Xin. Guozhen Xiao. Error correcting codes-- Theory and method[M].Xi'an: Xi'an Electronic and Science University press, 2006.

[4] Truong, T.K.Chen, P.D.Wang etc. Fast Transform for Decoding Both Errors and Erasures of Reed-Solomon Codes Over GF $\left(2^{\mathrm{m}}\right)[\mathrm{J}]$. IEEE Trans Communications.2006,vol.54(2):181-186.

[5] Pei Liu. Jianhua Ge. Gang Liu. Performance evaluation of the concatenated convolution codes [J]. Chinese Journal of Electronics, 2003,S1(07):1089-1091. 